\title{
Softwarekomponenten zur Meßdatenverarbeitung für experimentelle Untersuchungen an PTCA-Kathetern
}

\author{
Kraft M., Krebs, K., Rutsch W.*, Boenick U. \\ Institut für Mikrotechnik und Medizintechnik, Technische Universität Berlin \\ * Zentrum für Innere Medizin, Medizinische Klinik und Poliklinik I, Charité, Humboldt Universität Berlin
}

\section{EINLEITUNG}

Ein Prüfstand für Ballonkatheter (Herzkranzgefäßmodell) ist den anatomischen Verhältnissen nachempfunden. Meßbar sind Aktions- und Reaktionskräfte an verschiedenen Positionen ebenso wie Drücke im Ballon und in Modellstenosen [1]. Nachdem der grundlegende Aufbau der Meßdatenerfassung und Ansteuerung aktiver Prüfstandkomponenten [2] ebenso wie ein Fuzzy-Bewertungsprogramm [3] bereits vorgestellt wurden, liegen die Schwerpunkte dieses Beitrages auf der Beschreibung der zur Datenerfassung und Datenaufbereitung entwickelten Software.

\section{SOFTWAREKONZEPT}

Die Datenverarbeitung im Herzkranzgefäßmodell geschieht in drei verschiedenen Programmpaketen. Als Entwicklungsumgebung für die Datenerfassungssoftware wurde LabView ${ }^{\top M}$ der Fa. National Instruments gewählt. Durch Verwendung einer Datenerfassungskarte des gleichen Herstellers konnten Kompatibilitätsprobleme ausgeschlossen werden. Eine weitergehende Datenaufbereitung kann in Microsoft ${ }^{\oplus}$ Visual Basic for Applications $^{\mathrm{TM}}{ }^{\mathrm{M}}$ Programmen innerhalb von Microsoft $^{{ }^{\circ}}$ Excel $^{\mathrm{TM}}$ vorgenommen werden. Dieses Programmpaket kam aufgrund umfangreicher Programmiermöglichkeiten und seiner weiten Verbreitung zur Anwendung. Eine abschließende Verarbeitung der Meßdaten erfolgt im dritten Programmpaket, einem unter Borland ${ }^{\star}$ Delphi $^{\top \mathrm{M}}$ entwickelten Bewertungsprogramm [3].

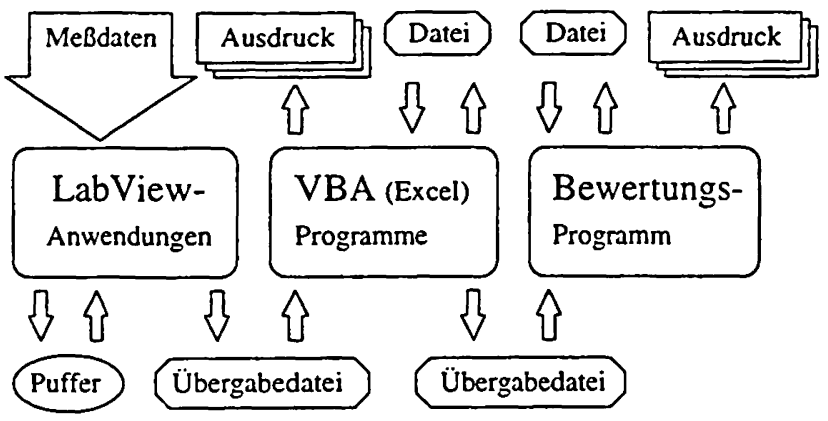

Abb. 1: Softwarekonzept des Prüfstandes

\section{DATENERFASSUNG MIT LABVIEW ${ }^{\text {TM }}$}

LabView $^{\mathrm{TM}}$ (Laboratory Virtual Instrument Engineering Workbench) ist ein symbolorientiertes Programmiersystem und verwendet eine rein graphische Programmiersprache. Jedes LabView ${ }^{\top \mathrm{T}}$-Programm besteht aus einer Frontblende, über die eine Bedienung des' Programmes möglich ist, und einem Blockdiagramm, welches den eigentlichen Quellcode enthält. Vordefinierte Objekte in unterschiedlichen Anwendungsbibliotheken und graphische Tools ermöglichen eine vorrangig am Datenflu $B$ orientierte Programmierung. Dieses Verdrahten von
Subroutinen an ihren Datenein- und ausgängen im Blockdiagramm stellt eine sehr effektive und leicht nachvollziehbare Art der Programmierung dar. Die Frontblenden zur Bedienung der entwickelten Programme imitieren durch Plazierung von Schaltern, Knöpfen und Anzeigen reale Meßgeräte auf dem Bildschirm, weshalb sie vom Hersteller als virtuelle Instrumente (VI) bezeichnet werden.

Nachteilig wirkt sich, wie in allen Programmen, eine mit Zunahme des Umfanges schnell verloren gehende Übersicht aus. Das Problem übereinanderliegender oder nicht mehr im Bildschirmbereich sichtbarer Sequenzen läßt sich jedoch durch konsequente Zusammenfassung von Routinen in eigenen Objekten etwas abschwächen.

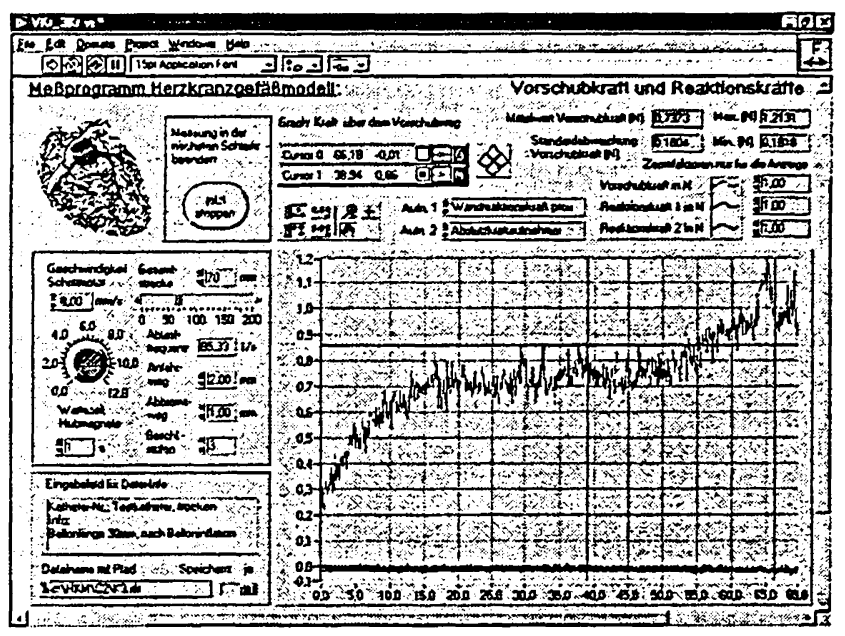

\section{Abb. 2: Frontblende eines LabView ${ }^{T M}$-Programmes}

Programme zur Meßdatenerfassung wurden für zahlreiche Untersuchungen an PTCA-Kathetern geschrieben. Sie beinhalten neben der Erfassung der jeweiligen Meßdaten (Kraft, Druck, Ballondurchmesser, digitale Inputs) immer auch die Ansteuerung der aktiven Prüfstandkomponenten (Schrittmotor, rotatorischer Antrieb, Hubmagnete an den Klemmen des Antriebes [siehe 2], Pumpenmotor, Magnetventil usw.).

Die Darstellung der Meßergebnisse auf dem Bildschirm dient zunächst nur der Beurteilung des Erfolgs der Messung. Alle Daten werden an Spreadsheet-Files übergeben, die neben selbst definierbaren Dateiinformationen, Datum und Uhrzeit der Messung auch Meßparameter wie Länge der Meßstrecke, verwendete Vorschubgeschwindigkeit, genutzte Aufnehmer und Kalibrierfaktoren enthält.

Das Ziel der weiteren Entwicklung der Datenerfassungsprogramme besteht neben einer Optimierung der Routinen und der Integration noch nicht realisierter Funktionen in der zentralen Ansteuerung aller Einzelmessungen aus einer Hauptroutine. 


\section{VBA-ANWENDUNGEN IN MS-EXCEL ${ }^{T M}$}

Ein entwickeltes VBA-Programm zur Meßdatenaufbereitung hat das Ziel, dem Benutzer möglichst einfach die Ergebnisse aus zuvor durchgeführten Katheter-Prüfungen darzustellen und einen Vergleich verschiedener Katheter zu ermöglichen. Das Programm stellt die Registrierblätter: Diagramme, Zusätze, beschreibende Statistik, analytische Statistik und Hilfe zur Verfügung.

Zunächst ist eine Kennzeichnung der Dateien notwendig, deren Meßdaten aufbereitet bzw. verglichen werden sollen. Die Auswahl erfolgt über Bezeichnung des jeweiligen Hauptgefäßes im Modell und der interessierenden Untersuchung. Daraufhin sind alle verfügbaren zugehörigen Dateien einlesbar und werden mit Namen und Speicherdatum angezeigt. Im folgenden Registrierblatt "Zusätze" können von der vordefinierten Beschriftung des Diagrammes (Titel entsprechend Untersuchung, Reihenbezeichnung entsprechend Dateiname usw.) abweichende Änderungen vorgenommen werden.

Im Ordner "Beschreibende Statistik" sind nachfolgende Optionen wählbar: Regressionsgerade in das Ergebnisdiagramm zeichnen, Geradengleichung der Regressionsgeraden angeben, Korrelationskoeffizienten bestimmen, Mittelwerte für Wertebereiche berechnen (Bildung gleich großer Wertebereiche für alle Meßreihen an beliebig vielen Stützstellen), arithmetisches oder geometrisches Mittel über die gesamte Reihe berechnen.

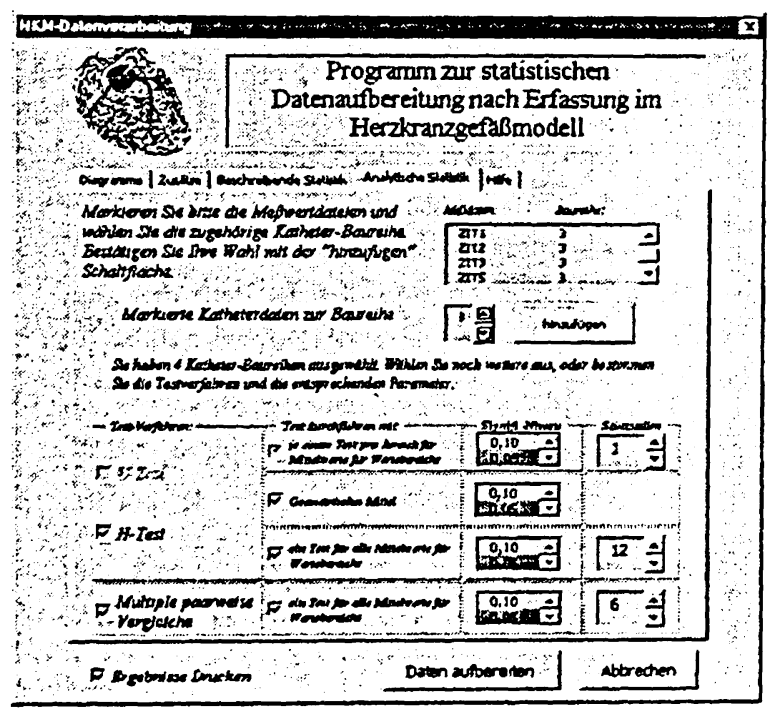

Abb. 3: Registrierblatt "Analytische Statistik" eines VBA-Programmes zur Datenaufbereitung

Das Registrierblatt "Analytische Statistik" (Abb. 3) dient der Bildung zu vergleichender Klassen (Baureihen) sowie der Auswahl statistischer Signifikanztests. Wählbar sind der U-Test als Rangsummentest für den Vergleich zweier Mittelwerte stetiger Verteilungen, der H-Test als Erweiterung des U-Testes auf mehr als zwei Mittelwerte und der Test "Multiple paarweise Vergleiche der mittleren Ränge", der sich nicht auf die zuvor definierten Katheterklassen (Baureihen), sondern auf dic Kathetermeßreihen bezieht.

Die Signifikanztests können in zwei unterschiedlichen Varianten durchgeführt werden, dic sich in der Art der verwendeten Mittelwerte unterscheiden. Einerscits ist es möglich, alle an der vorgegebenen Anzahl von Stützstellen gebildeten Mittelwerte einer Meßreihe bzw. Klasse (Baureihe) zusammen in den Test einzubeziehen. Andererseits werden Tests unter den Klassen (Baureihen) für jede zugeordnete Stützstelle anhand der Mittelwerte durchgeführt. Dies ist sinnvoll, wenn beispielsweise Vorschubkräfte in einer Stenose untersucht werden. Erst für die letzten, kurz vor der Verengung liegenden Stützstellen, sind hier signifikante Unterschiede zwischen Kathetern verschiedener Hersteller zu erwarten.

Die Anzahl der Stützstellen läßt sich beliebig erhöhen. Der Mindestwert, der vom jeweiligen Testverfahren und der Anzahl der Klassen abhängt, kann jedoch nicht unterschritten werden. Sind die Katheter-Baureihen festgelegt und die Signifikanztests ausgewählt, muß für jeden gewählten Test noch die Irrtumswahrscheinlichkeit und damit das Signifikanzniveau angegeben werden. Die Ausgabe der Testergebnisse, des Diagramms, berechneter Geradengleichungen usw. erfolgt in eine neue Ecxel ${ }^{T M}$. Arbeitsmappe.

Ein weiteres VBA-Programm dient der Erzeugung von Übergabedateien an ein Fuzzy-Bewertungsprogramm [3]. Einzelne in Tabellenblättern eingelesene Werte werden in einer entsprechenden Datei an der Stelle positioniert, die dem jeweiligen Meßwert zugeordnet ist.

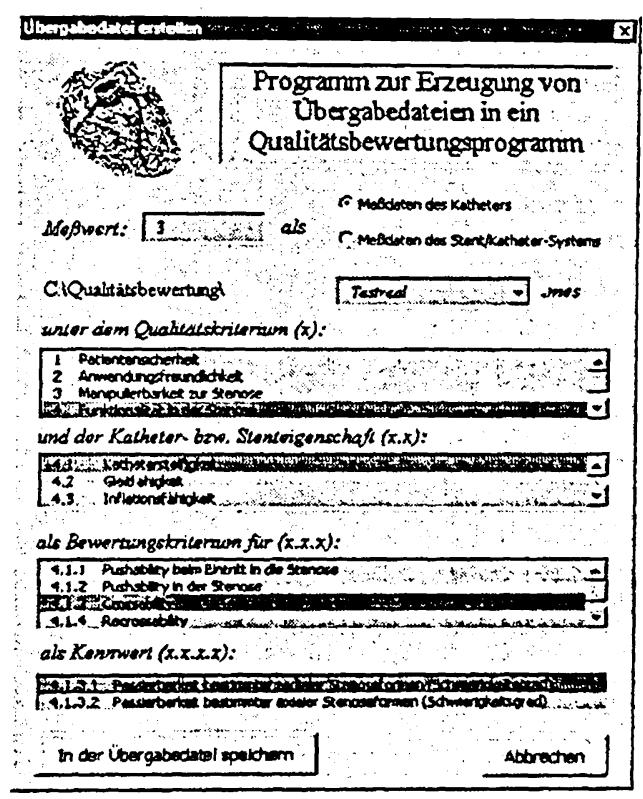

Abb. 4: VBA-Datenübergabeprogramm

\section{LITERATUR}

[1] Kraft, M.; Rutsch, W.; Boenick, U.: Ein multifunktionales Herzkranzgefäßmodell: Nutzungsvarianten für vergleichende Untersuchungen an PTCA-Kathetern. Zeitschrift für Biomed. Technik, Band 43 (Erg.band), 1998 [2] Kraft, M.; Wendt, O.; Rutsch, W.; Boenick, U.: Realisierung eines Katheterantriebes und der MeBwertcrfassung in einem Herzkranzgefäßmodell für PTCA. Katheter, Zeitschrift für Biomed. Technik, Band 42 (Erg.band), 1997, S. 484-485

[3] Kraft, M.; Marsolek, I.; Rutsch, W.; Bocnick U.: Ein Fuzzy-Bewertungsprogramm für PTCA-Katheter. Zcitschrift für Biomed. Technik, Band 42 (Erg.band). 1997. S. 439-442 